\title{
Understanding a Heterogeneous Catalyst in Action
}

\author{
Jeroen A. van Bokhoven*, Christiane Kartusch, and Shreekant Satav
}

\begin{abstract}
The role of catalysis in the clean production of energy and chemicals will become increasingly important and new catalysts must be developed. To improve the activity, selectivity, and stability of a heterogeneous catalyst, the electronic and geometric structure of the catalytically active site must be controlled. In situ characterization combined with kinetic analysis provides insights into the functioning of a heterogeneous catalyst. This aids the synthesis of a new generation of catalysts that converts new feedstocks, which are derived from sustainable sources, into energy and chemicals.
\end{abstract}

Keywords: Chemicals · Energy · Heterogeneous catalysis · In situ characterization · Sustainability

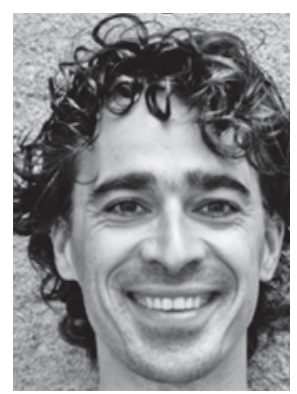

Jeroen van Bokhoven is SNF-Professor in Heterogeneous Catalysis at the Institute for Chemical and Bioengineering, ETH Zürich, since April 1st 2006. He was born December 22nd 1971 in Maassluis, The Netherlands. He finished his studies in 1995 in chemistry at Utrecht University (NL). He obtained his $\mathrm{PhD}$ with distinction in 2000 in inorganic chemistry and catalysis. From 1999 until 2002 he was head of the XAS (X-ray absorption spectroscopy) users-support group at Utrecht University. In 2002, he moved to the ETH, where he worked as senior researcher in the group of professor Prins. Van Bokhoven works in the field of heterogeneous catalysis and (X-ray) spectroscopy. Goal is the determination of structure-performance relationships, which aid the design and construction of better catalysts for cleaner and more efficient processes. His main interests are heterogeneous catalysts and developing advanced tools in X-ray spectroscopy to study the catalyst structure under catalytic relevant conditions. Their combination provides insight into the structure and function of the catalytically active sites.

\footnotetext{
${ }^{\star}$ Correspondence: Prof. Dr. J. A. van Bokhoven ETH Zürich

Institute for Chemical and Bioengineering

$\mathrm{HCl} \mathrm{E127}$

$\mathrm{CH}-8093$ Zürich

Tel.: +41446334372

Fax: + 41446321162

E-mail: j.a.vanbokhoven@chem.ethz.ch
}

\section{Introduction}

Never has there been a greater need to develop clean chemistry and technology for a sustainable society. The challenges that lay ahead are enormous and sometimes underestimated. We must develop processes that are environmentally friendly, non toxic, and have no harmful side effects, which operate under mild conditions and are energy-efficient. We must produce energy from fossil fuels in a more environmentally benign manner (for example by sequestering all $\mathrm{CO}_{2}$ and using clean technology) and/or we must switch from polluting fossil fuels and feedstocks to renewable sources such as biomass. Moreover, production of energy from alternative sources like water, wind, and the sun must be developed further in an economically viable manner.

Improvements must be made in many fields of science and technology and catalysis is an important field. The function of a catalyst is to increase the rate of a reaction without being consumed in the process. A selective rate enhancement produces a chemical in a highly selective manner. For this reason, catalysts have played an extremely important role in the chemical industry for the production of chemicals (bulk, intermediates, and fine chemicals) and in pollution control. About $80 \%$ of all products have been in contact at least once with a catalyst during the course of their production. The role of catalysts in the clean production of energy and chemicals and in pollution control will become even more important. Because of the different nature of the chemicals, new catalysts must be developed to convert a biomass feedstock to useful chemicals and for energy applications. ${ }^{[1]}$

Catalyst design requires in-depth understanding of the functioning of a cata- lyst. Because developments of new characterization methods over the last decade enable structural analysis under catalytically relevant conditions, our understanding of a working catalyst has increased considerably. ${ }^{[2]}$ Nonetheless, more often than not, the structure of a catalytically active site on a heterogeneous catalyst is unknown for the reaction of molecules more complex than ethylene. ${ }^{[3]}$ As a result, the design of new and better catalysts is still impossible.

We aim at the synthesis of catalysts that are structurally controlled on the atomic, nano, and meso scales and to develop and employ spectroscopic methods to determine the structures of catalytically active sites. Kinetic analysis of reaction networks reveals the kinetically important reaction steps, which must be controlled. A better understanding of the catalytic process from synthesis and activation to the actual reaction and catalyst deactivation is extremely important when designing a chemical process.

We work on zeolites, which are used in the valorization of alkanes in reactions such as cracking, alkylation, and selective oxidation. The latter reaction is one of the more challenging reactions in heterogeneous catalysis and will remain important because of the increasing production of hydrocarbons from biomass feedstock by gasification to $\mathrm{H}_{2} / \mathrm{CO}$ (syngas) and Fischer-Tropsch synthesis. Control of the pore topology and the introduction of mesoporosity are combined with the addition of transition metal ions to produce active centers with a well-defined structure. Supported metal catalysts, such as gold, palladium, platinum, and ruthenium, are used to oxidize and hydrogenate selectively. These reactions are relevant to the synthesis of useful chemicals from renewables and/or the use of clean technology. 
Renewables that are derived from biomass are a mixture of complex molecules, which contain many heteroatoms, notably oxygen. These molecules, such as glycerol, 2,5 furan dicarboxylic acid, aspartic acid, and levulinic acid, are to be converted into useful chemicals, for which new catalysts and processes must be developed. ${ }^{[1]}$ These molecules are (potential) feedstock for nylons, fuels, solvents, and polymers, etc. Some of the reactions to be developed are selective hydrogenation, oxidation, deoxygenation, and dehydration, and $\mathrm{C}-\mathrm{C}$ bond formation, such as aldol condensation. The chemical reaction network of such molecules, often under aqueous conditions, is hardly understood and represents a great scientific challenge.

We also investigate model reactions, such as the oxidation of $\mathrm{CO}$ and catalytic systems to determine the structures of catalytically active sites in a functioning catalyst. Catalyst characterization is essential to the understanding of how a catalyst functions. We develop new spectroscopic tools that enable the determination of the structure of the catalytically active site, an example of which is given below. These and additional methods are based on the use of X-rays together with other spectroscopic methods such as infrared and NMR spectroscopy.

\section{Catalysis and the Controlled Synthesis of Catalysts}

Reactants adsorb on the surface of a catalyst and, after reaction, they must desorb to produce the products and to free the catalyst to restart the catalytic cycle. Ideally, the surface of a catalyst is controlled at the various length scales. Because traditional wet synthesis fails to produce such surfaces, the emphasis is on alternative and new methods based on nanotechnology in the field of heterogeneous catalysis. ${ }^{[4,5]} \mathrm{We}$ aim to synthesize extremely well-defined structures in such a manner that the electronic and geometric structure of the catalytically active site is controlled. This will produce highly selective catalysts and, at the same time, opens up new possibilities for the in situ characterization of the structure of the catalytic site.

To provide an in-depth example of catalytic synthesis, we control the surface of a catalyst by means of atomic layer deposition (ALD) of metal precursors on a silica support to enable the synthesis of metal oxide surfaces, which are controlled in terms of number of layers and atomic composition of each layer. ALD is based on the sequential application of self-limiting gas-solid reactions. ${ }^{[6]}$ The ALD process consists of four steps within one cycle. During the first step, the gaseous precursor material is admitted to the substrate surface. The precursor chemisorbs and a partial but surface-saturated monolayer of the precursor is formed on the substrate. After removal of the excess precursor by an inert gas purge, the second precursor is introduced, which reacts to produce the desired material. After a finished cycle the process can be repeated until the desired number of layers is reached. This enables the control of the chemistry that occurs on the surface. To produce supported metal catalysts, size-controlled metal particles can be deposited. Near mono-disperse particle size distributions can be obtained by synthesizing colloidal particles. Ordered mesoporous silica (SBA-15) (Fig. 1), which has an extremely large surface area is used as the backbone of the support for depositing the metal oxides. This produces surface areas that by far surpass those obtained through synthesis of nano-sized or mesoporous metal oxide particles. Another major advantage is that all the metal oxide is on the surface, and bulk spectroscopic methods can be used to determine its structure. Such synthesis methods will enable

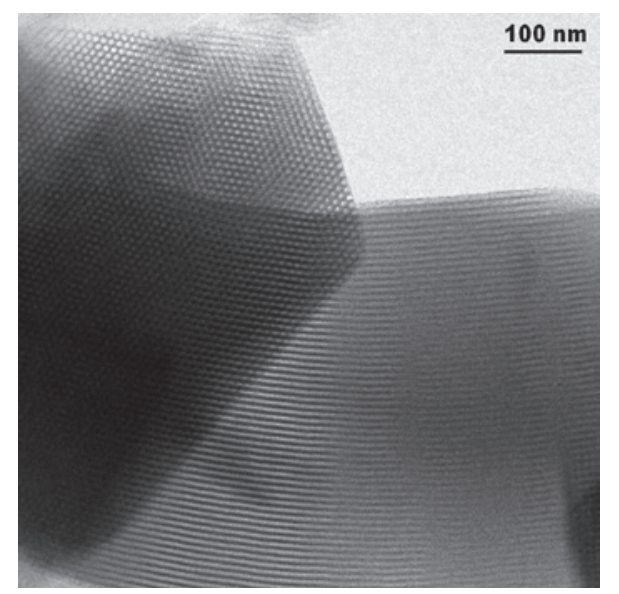

Fig. 1. Siliceous mesoporous SBA-15. The mesopores are hexagonally oriented, shown by the leftmost crystal, where one is looking down into the pores. The surface area of this sample is $926 \mathrm{~m}^{2} / \mathrm{g}$. us to control the chemistry that such catalytic surfaces display.

\section{Gold catalysts: Why is it Important to Control Particle Size? Manipulation of the Selectivity in Hydrogenation of $\alpha, \beta$-Unsaturated Aldehydes}

The performance of supported metal catalysts is clearly dependent on the type of metal, the support, the presence of other metal atoms, and the particle size. ${ }^{[7]}$ Gold catalysts are probably the best example of catalyst performance affected by its size. Solid gold is the most inert of all metals: ${ }^{[8]}$ however, when deposited on an oxide support as nano-sized particles, it is highly active for many reactions, such as oxidation and hydrogenation. ${ }^{[9]}$ Many of these reactions have the potential to develop to new applications for catalysis by gold in pollution control, sensors, chemical processing, and fuel cell technology. We have determined the performance of nano-sized gold, supported on alumina, in the hydrogenation of cinnemaldehyde. ${ }^{[10]}$ The selective hydrogenation of $\alpha, \beta$-unsaturated aldehydes is a critical step in the production of fine chemicals, such as flavor and fragrance compounds and pharmaceuticals. Cinnamaldehyde is generally used as a probe molecule to elucidate the selectivity in the hydrogenation of unsaturated aldehydes. The unsaturated alcohol is the desired product, but over most unpromoted supported metals, saturated aldehyde is selectively formed (Scheme). Since gold interacts weakly with reactants, intermediates, and products, in contrast to other transition metals, it is a good candidate for selective hydrogenation of molecules with a multiple of functional groups.

Fig. 2 (left) shows that the rate of hydrogenation per gram of gold in the catalyst increased with decreasing particle size. The smaller the particles, the higher

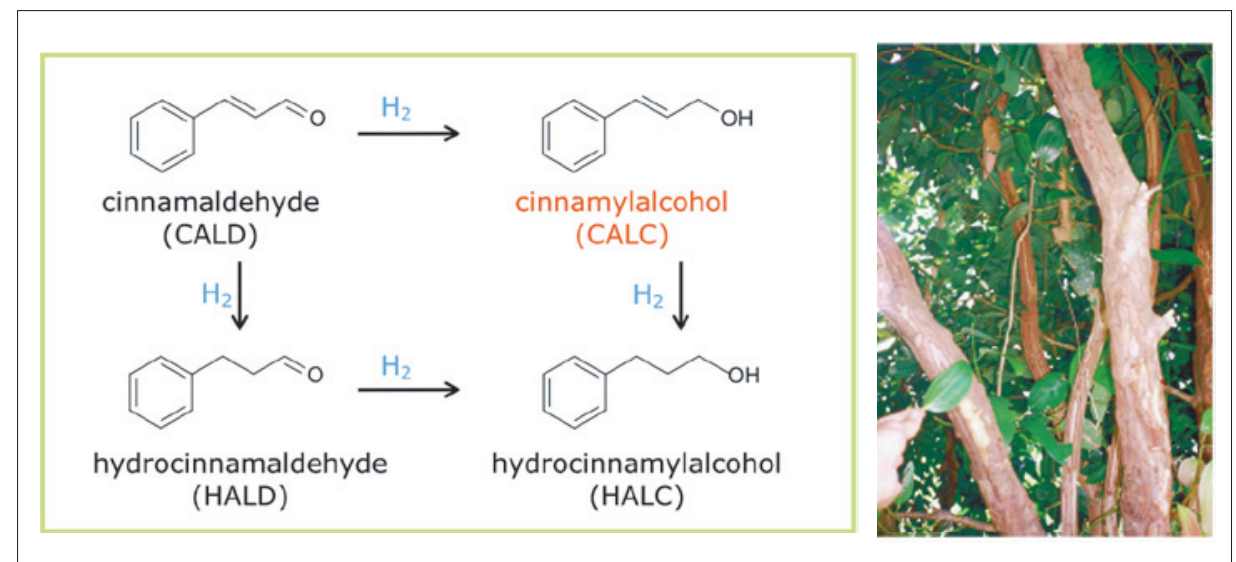

Scheme. (Left) Hydrogenation of the unsaturated aldehyde cinnamaldehyde. (Right) The cinnamon tree is the major source of cinnamon. The oil of its bark contains about $90 \%$ cinnamaldehyde. 
was the rate. Fig. 2 (right) indicates that the turnover frequency (TOF), calculated as the number of gold atoms that adsorb hydrogen, increased with decreasing particle size. In addition to increasing TOF, the smaller particles were more selective to the desired unsaturated alcohol (see the numbers near the points). In contrast, the TOF for cyclohexene hydrogenation did not depend on particle size. This indicates that the smaller particles are more reactive toward hydrogenation of the aldehyde bond. The chemistry of gold particles is size-dependent. The origin of this phenomenon lies in the oxophilic nature of smaller particles, caused by a change in the electronic structure. ${ }^{[11-13]}$ Using in situ $\mathrm{X}$ ray absorption spectroscopy, a dependence of the geometric and electronic structures of catalytically active gold on particle size was established. ${ }^{[14]}$ The interaction of reactants with a transition metal depends on the energy of the $\mathrm{d}$ band, which is also related to its filling. Bonding of reactants to the surface generates bonding and anti-bonding states. Solid gold is inert, because the anti-bonding state is filled. However, because of the coordinative unsaturation of nano-sized particles, the $\mathrm{d}$ band narrows, causing it to shift toward the Fermi level, thus making it more reactive. ${ }^{[8,12,15]}$ This pushes the anti-bonding state, formed by the interaction of gold and oxygen, hydrogen, and carbon monoxide, to above the Fermi level, as revealed by XAS. Because the surface of solid gold does not interact with most reactants and its surface remains empty, it is an unsuitable catalyst; in contrast, nano-sized gold weakly adsorbs various reactants, making it ideal for catalysis.

This example illustrates the potential of nano-sized gold to selectively catalyze reactions at reasonable rates. Nano-sized gold is active in many reactions, of which the activity and selectivity in oxidation reactions, such as oxidation of $\mathrm{CO}$, alkanes, and alcohols are the most studied.

\section{Light and the Study of Catalysts}

Understanding the functioning of a catalyst requires a detailed knowledge of its structure. The life cycle of a catalyst can be (arbitrarily) divided into four parts: synthesis, conditioning, functioning, and deactivation. A better understanding of one or all of these four parts enables the improvement of the catalyst. There are many methods that lead to insight into the structure of a catalyst and into the processes that occur during synthesis, conditioning, functioning, and deactivation. Different parts of the spectrum of light are used in spectroscopic and scattering methods, which can be used to study catalysts. NMR, which employs radio frequencies, infrared, UVvis, and Xray diffraction and absorption are used. We

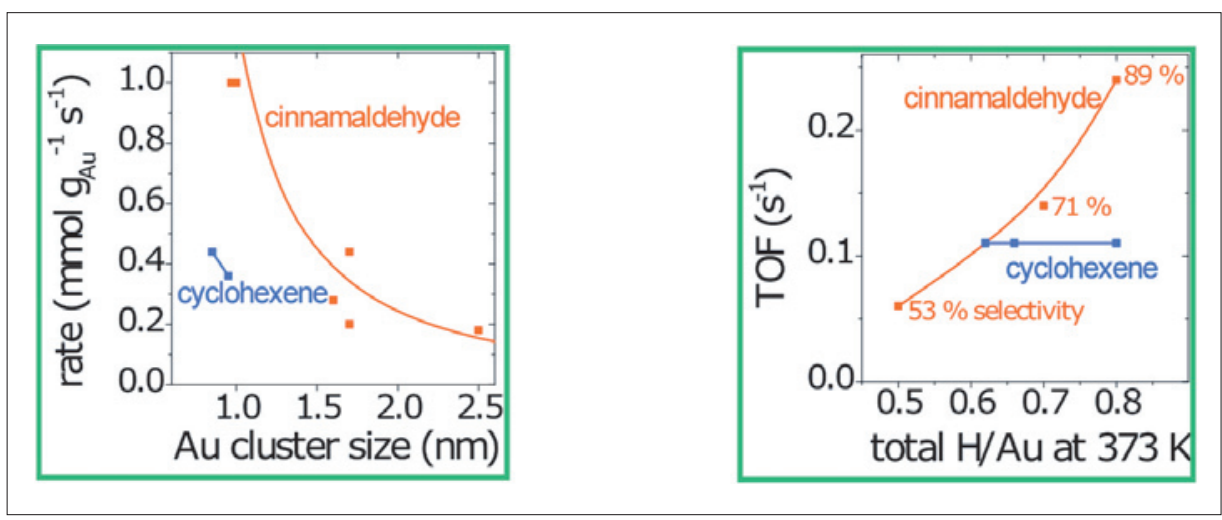

Fig. 2. (Left) Rate of hydrogenation of cinnemaldehyde as function of gold particle size. (Right) The corresponding TOF, calculated as the number of gold atoms that adsorb hydrogen. The numbers near the curve give the selectivity to the desired unsaturated alcohol (reaction conditions: $\left.8 \mathrm{MPa} \mathrm{H}_{2}, 373 \mathrm{~K}\right)$.

apply these methods to elucidate the structure of catalytically active sites, to detect reactants and intermediates on the catalyst surface, and to detect the interaction between these and the active site.

\section{Shining Light on a Working Catalyst: Structure of a Zeolitic Active Site}

Zeolites are silica-alumina microporous materials, which function as catalysts in many reactions such as cracking, isomerization, acylation, alkylation, Meerwein-Ponndorf-Verley (MPV) reduction, and Baeyer-Villiger oxidation. One of their unique properties is their pore system, which enables us to tune the selectivity of a reaction by size exclusion of reactants, intermediates, and/or products. In situ X-ray absorption spectroscopy at the $\mathrm{Al} \mathrm{K}$ edge reveals the structure of the catalytically active site during catalyst performance. ${ }^{[16]}$ The Brønsted acid active site is a hydroxyl group that bridges an aluminum atom to a silicon atom (Fig. 3). Both aluminum and silicon are tetrahedrally coordinated. Extended X-ray absorption fine structure (EXAFS) analysis at the Al K edge under reaction conditions ${ }^{[17]}$ showed that the aluminum tetrahedron is heavily distorted because of the long aluminumhydroxide bond (Table). However, with the addition of ethylene, which reacts to form a hydrocarbon intermediate adsorbed on the active site, distortion occurred to a lesser extent. When the temperature was increased, the long bond was restored because of desorption of the intermediate. An active site of a heterogeneous catalyst, such as the one in a zeolite, shows dynamical changes in its structure to accommodate reactants and intermediates enabling catalysis to occur. A heterogeneous catalyst is a dynamic structure and the dynamics must be determined to learn more about its functioning. [18]

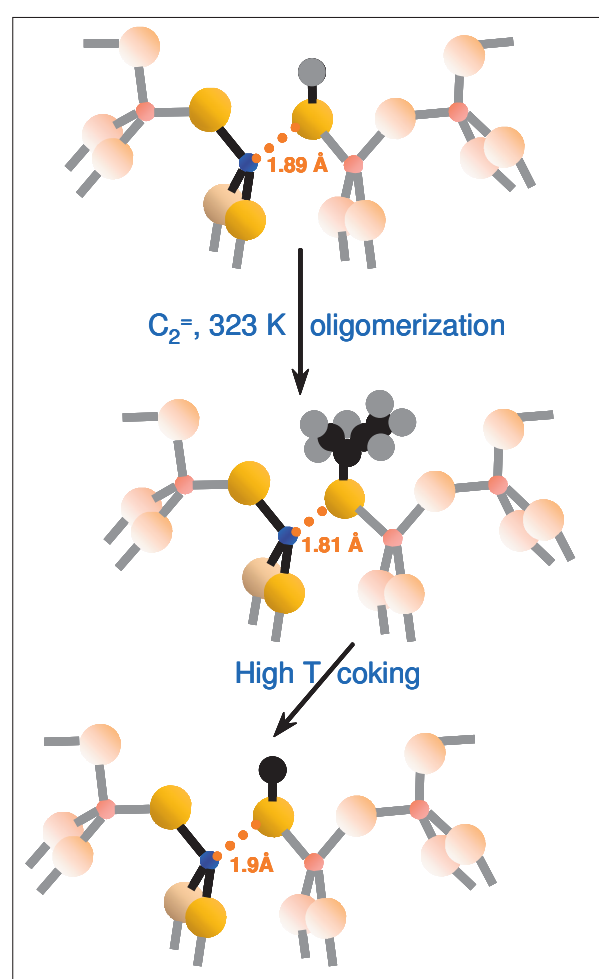

Fig. 3. The dynamical changes in the local structure of a catalytically active site in a situ Al K edge EXAFS analysis. Blue: aluminum, red: silicon, yellow/brown: oxygen, grey: hydrogen, and black: carbon.

Table. Dynamical changes in the local structure of the active center in a zeolite under reaction conditions.

$\begin{array}{lll}\text { sample } & \begin{array}{l}\text { Al-O } \\ \text { coordination }\end{array} & \begin{array}{l}\text { Bond length } \\ [\AA]]\end{array} \\ \mathrm{HY} & 3 & 1.66 \\ \mathrm{HY}\left(\mathrm{C}_{2}=\right) 323 \mathrm{~K}^{\mathrm{a}} & 3 & 1.89 \\ & 1 & 1.64 \\ \mathrm{HY}\left(\mathrm{C}_{2}=625 \mathrm{~K}^{\mathrm{b}}\right. & 3 & 1.81 \\ & 1 & 1.65 \\ & 1.91\end{array}$

${ }^{a}$ exposing the active site to $5 \%$ ethylene in helium at $323 \mathrm{~K}$; bafter heating to $625 \mathrm{~K}$. zeolite under reaction conditions, based on in 


\section{Conclusion}

It is urgent that we develop new catalysts to meet the challenge of developing a sustainable society. Our approach is to synthesize well-defined catalysts to enable control of their structure on atomic, nano, and meso scales and to implement in situ characterization and kinetic analysis. Our aim is to understand how heterogeneous catalysts function to enable us to improve the activity, selectivity, and stability of a catalytic process.

\section{Acknowledgement}

The Swiss National Science Foundation is thanked for financial support-

Received: February 2, 2009

[1] A. Aden, J. Bozell, J. Holladay, J. White, A. Manheim, 'Top Value Added Chemical from Biomass' Pacific Northwest National Laboratory and National Renewable Energy Laboratory, Richland, WA, 2004.

[2] J. M. Thomas, Angew. Chem, Int. Ed. 1994, 33, 913.

[3] G. A. Somorjai, A. L. Marsh, Phil. Trans. Royal Soc. 2005, A 363, 879 .
[4] J. M. Thomas, R. Raja, D. W. Lewis, Angew. Chem., Int. Ed. 2005, 44, 6456.

[5] G. A. Somorjai, J. Phys. Chem. 1990, 94, 1013.

[6] R. L. Puurunena, J. Appl. Phys. 2005, 97, 121301.

[7] B. C. Gates, Chem. Rev. 1995, 95, 511.

[8] B. Hammer, J. K. Nørskov, Nature 1995, 376, 238.

[9] G. C. Bond, D. T. Thompson, Catal. Rev. Sci. Eng. 1999, 41, 319.

[10] E. Bus, R. Prins, J. A. van Bokhoven, Cat. Comm. 2007, 8, 1397.

[11] J. T. Miller, A. J. Kropf, Y. Zha, J. R. Regalbuto, L. Delannoy, C. Louis, E. Bus, N. Weiher, J. A. van Bokhoven, J. Catal. 2006, 240, 222.

[12] J. A. van Bokhoven, J. T. Miller, J. Phys. Chem. C 2007, 111, 9245.

[13] H. Falsig, B. Hvolbaek, I. S. Kristensen, T. Jiang, T. Bligaard, C. H. Christensen, J. K. Nørskov, Angew. Chem., Int. Ed. 2008, 47, 4835.

[14] J. A. Van Bokhoven, Chimia 2009, in press.

[15] J. A.van Bokhoven, C. Louis, J. T. Miller, M. Tromp, O. V. Safonova, P. Glatzel, Angew. Chem., Int. Ed. 2006, 45, 4651.

[16] A. M. J. van der Eerden, J. A. van Bokhoven, A. D. Smith, D. C. Koningsberger, Rev. Sci. Instrum. 2000, 71, 3260.

[17] J. A. van Bokhoven, A. M. J. van der Eerden, R. Prins, J. Am. Chem. Soc. 2004, 126, 4506.

[18] J. Singh, E. M. Alayon, M. Tromp, O. V. Safonova, P. Glatzel, M. Nachtegaal, R. Frahm, J. A. van Bokhoven, Angew. Chem., Int. Ed. 2008, 47, 9260 . 